Review

\title{
Spirituality and Quaker Approaches to Substance Use and Addiction
}

\section{Helena Chambers}

Director of Quaker Action on Alcohol and Drugs, 21 Church Street, Tewkesbury GL20 5PD, UK; E-Mail: helenaqaad@hotmail.com; Tel.: +44-1684-299-247

Academic Editors: Chris Cook and Wendy Dossett

Received: 16 February 2015 / Accepted: 30 March 2015 / Published: 8 April 2015

\begin{abstract}
The Religious Society of Friends (Quakers) has held a consistent testimony of abstinence and moderation regarding alcohol and other substances. This paper outlines the historical background, and describes modern Quaker understandings of moderation. It then outlines hitherto unpublished results regarding spirituality from the only study to date about Quaker behaviour and atttitudes regarding substance use. The association between low substance use and religiosity is established in the literature, but the role of spirituality is less defined. This study methodology allowed an unusually detailed analysis of different aspects of spirituality. Results generally support Miller's suggestion that idiographic spirituality may have a role in resilience to higher substance use. However, spiritual practice through prayer/meditation emerges as having a more consistent role in the Quaker sample - a finding that is of interest and potential significance in considerations of resilience and recovery. The community dimension of shared spiritual attitudes towards substance use, and the spiritual values that underlie the interpretation of testimony, are also explored. The congruence that some Quakers find with the spiritual approaches of Alcoholics Anonymous is also discussed. It is concluded that spirituality is a significant factor in a Quaker balance that can mitigate immoderate use and support recovery from addiction, without, in general, excluding those who use at higher levels.
\end{abstract}

Keywords: spirituality; religion; Quaker; alcohol; substance use; addiction; liberal belief culture 


\section{Introduction}

\subsection{Background to the Quaker Testimony on Abstinence and Moderation}

The Religious Society of Friends (Quakers) was formed in the revolutionary England of the mid-seventeenth century. As a radical Protestant sect sharing some common origins with Puritanism, it displayed caution toward the injudicious or recreational use of alcohol from its earliest period. Quakerism's founder, George Fox (1624-1691), considered this to be incompatible with being a "man of religion" and left when pressed in company to drink more alcohol than was sufficient to quench his thirst [1]. However, George Fox was not abstinent; the water supply was uncertain and "small beer" was the normal and the safer drink at the time [2].

George Fox's guarded attitude to consumption was continued by eighteenth century Quakerism.

As temperance and moderation are virtues proceeding from true religion...we beseech all to be careful of their conduct and behaviour, abstaining from every appearance of evil; and excess in drinking has been too prevalent among many of the inhabitants of these nations, we commend to all Friends a watchful care over themselves, attended with a religious and prudent zeal against a practice so dishonourable and pernicious. (Yearly Meeting in London 1751 [3], 20.38).

During the nineteenth century, Quakers responded to the effects of cheap alcohol on the urbanised poor by becoming prominent in the (largely Non-Conformist) Temperance Movement. The term "temperance" was then used in its derived meaning of abstinence, rather than moderate usage. In later years, this general group identification of Quakers with total abstinence waned, particularly during the latter half of the twentieth century. Advice on substance use is now found in a section of Quaker Faith and Practice that is headed "abstinence and moderation", thus uniting both meanings of "temperance" in the modern Quaker approach:

Many yearly meetings hold very strong testimonies against any use of tobacco or alcohol. Within Britain Yearly Meeting some Friends advocate total abstinence from alcohol, others counsel moderation. Those who smoke tobacco, drink alcohol or abuse other substances risk damage to their own health, and may hurt or endanger other people. Such use can deaden a person's sensitivity and response to others and to God. Consider whether you should avoid these products altogether, discourage their use in others, especially young people, and refrain from any share in their manufacture or sale. Maintain your own integrity and do not let social pressures influence your decisions. ([3], 20.40).

This framing of substance use as a spiritual matter, and the need to resist social pressure when necessary, continue the threads first outlined by George Fox.

\subsection{Quaker Approaches to Addiction}

The concept of addiction or dependency as we understand it today did not develop until the nineteenth and twentieth centuries. George Fox did not focus specifically on addiction as regards 
alcohol or other substances. However, he does write more generally about any preoccupation that can gain ascendancy, and construes it as something that needs a profoundly spiritual response:

Friends, whatever ye are addicted to, the tempter will come in that thing: and when he can trouble you, then he gets advantage over you, and then you are gone. Stand still in that which is pure, and after ye see yourselves, and then mercy comes in. After thou seest thy thoughts, and thy temptations, do not think, but submit; and then power comes. Stand still in that which shows and discovers; and then doth strength immediately come. And stand still in the Light, and submit to it, and the other will be hushed and gone; and then content comes. ([3], 20.42).

To the modern eye, this is surprisingly consistent with current understandings of dependency-for example, with Orford's work "Excessive Appetites" which outlined the common features between different kinds of addictions [4]. It is also highly sympathetic with the framing of addiction as a "spiritual disease" by Alcoholics Anonymous, and with the idea that surrender ("submit") is a primary step. "After thou seest thy thoughts and thy temptations" can readily be construed within the "moral inventory" of the 12 steps, as well as within a cognitive-behavioural framework, and it chimes also with the more recent academic studies into addiction and spirituality [5].

A modern passage in Quaker Faith and Practice puts many of these frameworks together, recognises the difficulty of abstinence and moderation once dependency has taken hold, and considers how the Quaker community can respond:

For those trapped in substance abuse, such advice [as in $20.40^{1}$ ] may seem hollow. Commonalities exist between addictive behaviours with these substances and other compulsive actions such as in the areas of eating disorders, gambling, overwork and physical abuse. The causes go deep and may not be fully understood, but the resulting pain, fear, desperation and denial, damaging the abuser and all around that person, need to be supportively recognised. A meeting community should be ready to listen non-judgmentally, offer information about sources of help, refuse to enable people to continue in harmful patterns, and continue to offer an environment free from addictive practices. ([3], 20.41).

The "environment" in the last passage refers in part to the fact that Quaker Meeting Houses do not generally allow alcohol on the premises. This makes them a safe and spiritual space for Quakers, and it has also helped enable a tradition that many Meeting Houses are hired by Alcoholics Anonymous or one of the other Fellowships for their own meetings. Though this means no formal connection other than an arrangement to hire the room, there are examples known to the author of Fellowship members coming to Quakerism after first becoming familiar with a Quaker Meeting House in this way.

\subsection{Abstinence and Moderation as Quaker Testimony}

Abstinence and moderation as regards the use of alcohol and other substances forms a part of the broader Quaker testimony of simplicity. "Testimony" is a Quaker term that describes "corporate witness", when Quakers act collectively in harmony on an issue in a way that expresses a fundamental

\footnotetext{
1 See passage 20.40 on moderation, above.
} 
Quaker value [6]. However, Quaker testimony is not construed in the way that a doctrine or an authoritative text might be.

The testimony acts as a guideline that encourages spiritual reflection on personal action, rather than an injunction that provides strict rules. This emphasis non-doctrinal approach has been an integral part of Quakerism since its foundation. Even during the Temperance Movement of the nineteenth century, the Victorian Quakers noted that abstinence needs to arise from inner conviction inspired by God or spirit, rather than from obedience to an outer standard.

"the adoption of...abstinence as a rule, or even as a strong advice, is far from being desirable... it should derive from personal conviction..." [7].

This focus - and this balance between the testimony and personal reflection - has been carried forward into contemporary Quakerism. The "Advices" part of Quaker Faith and Practice suggests that each Friend has the responsibility to "consider their use":

In view of the harm done by the use of alcohol, tobacco and other habit-forming drugs, consider whether you should limit your use of them or refrain from using them altogether. Remember that any use of alcohol or drugs may impair judgment and put both the user and others in danger ([3], 1.02.40).

Alongside this focus on the individual path, Quakerism sees the "inner light" as being present in all people, and this spiritual value makes it inherently resistant to judgments of others. The general spirit of Quakerism's approach is expressed in this passage that comes at the beginning of Quaker Faith and Practice, and is held dear within the community:

Dearly beloved Friends, these things we do not lay upon you as a rule or form to walk by, but that all, with the measure of light which is pure and holy, may be guided; and so in the light walking and abiding, these may be fulfilled in the Spirit, not from the letter, for the letter killeth, but the Spirit giveth life.

Postscript to an epistle to "the brethren in the north" issued by a meeting of elders at Balby, 1656. ([3]: 1.01).

\subsection{Context of the Quaker Study}

The charity Quaker Action on Alcohol and Drugs (QAAD), which began as a temperance body in the nineteenth century, had a period of reflection at the end of the twentieth, when it discerned that to be relevant to modern Quakers it needed to espouse both abstinence and moderation. It sensed that the history of abstinence might have resulted in an assumption that Quakers did not suffer from problems with alcohol or other substances - but it also knew that no group was immune from these, or from addiction. Trustees felt that if Quakers were experiencing problems, they might feel a sense of isolation or shame. They appointed a worker with a background in addiction treatment (the present author) to investigate and to encourage Friends to reach out. The study that follows was part of that investigation, which was conducted in the early to mid 2000s, and the comments that are incorporated in this paper arise from my subsequent observations and experiences as a worker among Friends. 


\subsection{Theoretical Context for the Study}

QAAD's sense that addiction or problem use might be hard for Quakers to admit to has a foundation in the research literature. There is a strong research base relating to religious groups and how their beliefs and practices on alcohol and other substances relate to behaviour. In general terms, religiosity is associated with lower levels of substance use and substance problems [8-12]. The association has been particularly strongly observed in Protestant groups that enjoin abstinence/moderation through text or a tradition of witness [12-14]. It has therefore been suggested that religiosity may have a protective effect as regards the development of substance use and substance problems. Cook [15] summarises:

Various measures of religious behaviour, religious affiliation or "religiosity" are inversely correlated with substance use and misuse ([16], pp. 189-97; [17], pp. 169-72; [18], pp. 109-12). There is debate about how this effect may operate. For example, affiliation with a faith community may instil moral values which guard against substance use- especially illicit substance use-or substance misuse. However, religion is also associated with various measures of mental well-being ([16], pp. 118-37; [18], pp. 43-81) and may reduce substance misuse by improving coping skills or reducing perceived stress. On the other hand, the effect may simply be a function of conforming to the norms of a social group in which substance use or misuse is less acceptable.

In addition to the protective effect of religiosity on substance use, the association between lower use and higher engagement may also be mediated through the alienation of higher users-research literature has suggested that adherents of proscriptive groups may feel unable to remain within them if their substance using behaviour is incongruent with denominational norms [19].

It is certainly possible that this Protestant low use/religiosity link may exist amongst Quakers; however, the unique features of Quakerism may act to modulate this relationship. Quakerism's history is that of a Protestant group, but it is non-credal, and has a "liberal belief culture" that is resistant to external authority and behaviour that comes from adherence rather than spirit [20,21]. This lack of emphasis on doctrine, coupled with the non-judgmental spirit of Quakerism, may act to weaken the typical use/religiosity relationship both in terms of a lack of reinforcement of low use and a lack of alienation of higher users.

The place that spirituality and spiritual life might play in the religiosity/use relationship has been relatively little explored [12], especially among non-treatment adult populations. An additional consideration is that spirituality is increasingly considered as an overarching concept that can either be a part of religiosity, or distinct from it [17]. Miller considered that this distinction may be important as regards substance use, suggesting that "spirituality is fundamentally an idiographic aspect of the person" [5], whereas religiosity is more a social phenomenon, which would therefore be likely to have effects on substance using behaviours through psycho-social processes.

Given the potential importance of spirituality in the religiosity/use relationship, and the primacy that spiritual life has for Quakers, it was felt important to give it a strong focus in the study, and to do so in a way that allowed spirituality to be considered separately from other aspects of Quaker engagement. 
The study findings on spirituality have not hitherto been described. These make an original contribution to the field in terms of Quakers as a group, but also have implications for other religious groups and potentially for the study of substance problems and addiction. The findings on spirituality are outlined here. So too are the findings relating to how the testimony on abstinence and moderation is construed and lived by modern Quakers. These are discussed with particular reference to the workings of the Quaker community in promoting resilience to, or assisting recovery from, substance problems.

\section{Methodology}

\subsection{Research Design and Theoretical Framework}

The research questions discussed above were formulated into a single null hypothesis: that "there would be no relationship between engagement in Quakerism and substance using behaviours". The methodology devised to investigate this included both quantitative and qualitative methods.

The quantitative investigation involved measuring Quaker religiosity, and the use of various substances. The two elements were then correlated statistically to see if any relationships emerged.

The qualitative element of the study involved several open-ended items in a questionnaire that was sent to the whole sample, and further detailed exploration of theory and inductive categories through semi-structured interviews. Qualitative data is particularly important given that Quakers are a group without ministers or priests. Individual experience and perspectives carry a particular authority for Friends, and needed to be given the freest expression possible in the study, a consideration that also applied to the community dimension of the investigation. Qualitative data was also considered an indispensable component of the spirituality investigation.

The limitations on cross-sectional studies as regards establishing causality or the direction of it were acknowledged [22]. The methodology enabled the quantitative data to establish patterns of association, while the qualitative element was triangulated with it, and this allowed inductive themes to emerge. The study design of research was thus designed for "complimentarity" using Hammersely's categorisation of multi-strategy research [23].

Reference group theory, deviance theory, and psycho-social perspectives have all been used to explain behavioural conformity to normative religious standards. These theories were applied to the investigation of religiosity, and were used to construct the categories in the qualitative investigation of Quaker understandings of moderation.

However, theories on the role of spirituality as regards substance use were less well-developed, and it was also felt a more open approach was needed because of the Quaker approach to the nature of spirituality itself. For these reasons, the qualitative, and to a degree, the quantitative investigation of spirituality adopted a more inductive, theory-generating approach [24].

\subsection{Study Sample}

A total of 159 Quakers co-operated with the study and filled in the questionnaire, of whom 13 were selected for personal interview (with willingness to participate being one of the items on the questionnaire). The individuals involved came from five local Quaker Meetings which agreed to take part in the study. Stratification criteria were that these should cover a range of geographical locations over England, 
Wales and Scotland, a combination of rural and urban areas, and Meetings of different sizes. This method was chosen in preference to random sampling because the theories being tested included reference group theory.

Young Friends General Meeting (YFGM), a group consisting of Quakers between the ages of 18 and 35, also took part, to whom the same consideration applied, as this was likely to be the most significant reference group for these individuals. A total of 57 participants were in this group, and for some analyses this younger sample was considered separately.

Interview participants were selected from a range of ages and places, and to cover the full spectrum of substance use from higher levels to abstinence, and to cover a range of ages and Meetings. Typical and non-typical subjects were also chosen, which allowed a consideration of deviancy theory within the larger framework of reference theory. There was thus an element of theoretical sampling in the study.

\subsection{Quantitative Measures Used in the Study}

\subsubsection{Substance Use}

Respondents were asked about several substances including alcohol, nicotine and cannabis. Alcohol was measured by frequency of use and also by "most alcohol consumed on a single day in the last month", which was coded to provide a basic index of moderation.

\subsubsection{Quaker Engagement}

The measurement tools used to assess Quaker engagement drew on previous research which identified different aspects or dimensions of religiosity [25-27] in relation to substance use. The measurements used were sorted into three groups, which were shown by factor analysis to be statistically separable. The categories were:

- "formal involvement" (consisting of membership of the Religious Society of Friends vs. non-membership, frequency of attendance at Meeting for Worship, and a subjective measure of importance of Quakerism to the individual);

- "group identification measures" (measured by three Likert scale questions relating to feelings of affinity and closeness to other Quakers);

- "spirituality" consisting of: a scaled question about the frequency of private prayer/meditation; and levels of agreement with two Likert-scaled statements "spirituality is very important to me in my life" and "God's presence is available to me in everyday life".

These items cover six of the eight elements listed by Chitwood et al. [12] as having been used in substance use/religiosity studies (namely, organisational religiosity; religious affiliation; subjective religiosity; non-organisational religiosity, spirituality; and multi-dimensionality). Although "belief" questions were asked, they were not included in the statistical investigation, because Quakerism is not credal, and "God" is variously defined.

The measures of religiosity used are also consistent with those summarised by Allen and Lo [28]: 
Common measures of religiosity include denominational identification, frequency of participation in religious services, the degree of religion's meaningfulness to an individual, and the degree of the individual's closeness to members of a religious group ([12,29-31]).

The three spirituality indicators used in the Quaker study were partly derived from Hilty [27] who had used them with the Mennonites (a group that has some points of similarity with Quakers), and partly from Dandelion's earlier measures, which had been validated in a UK Quaker setting, and were sensitive linguistically and in belief terms to Quaker framings [20,21]. The frequency of "prayer or meditation" in particular was worded to take account of the fact that not all Quakers use the term "God" and may therefore not have been comfortable with the word "prayer". However, the "God's presence is available to me in everyday life", statement was (against expectation) included in the statistical analysis because it was filled in by both those who used the term "God" and those who used other language, such as "spirit", even though it was an optional item. This last item also relates closely to the "presence of God" item on the Daily Spiritual Experience Scale [32,33].

\subsubsection{Statistical Method Applied to the Quantitative Engagement Measures}

Many previous studies into substance use and religiosity have combined several measures of religious engagement into a single conglomerate "religiosity" score which is then correlated with substance use (e.g., Bock et al. [14]). However, the Quaker study analysed the three engagement elements individually, and correlated them separately with substance use. This remains unusual in the literature: Chitwood et al, for example, note that 12 out of the 105 studies they reviewed utilized both one-dimensional and multidimensional measures of religiosity [12].

This methodology allowed a close-grained interpretation, and, most significantly in view of the limited evidence-base, allowed spirituality to be considered separately.

Correlations were registered at the 0.05 level using Spearman's rho; these were adjusted using the Bonferroni correction to account for the inclusion of several factors. The level of stringency was therefore high, meaning that associations revealed were robust.

\subsection{Qualitative Methods}

All respondents completed a questionnaire that was given out and collected after Sunday Meeting for Worship on an agreed day. Pre-coded questions for the statistical analysis were widely supplemented by open-ended qualitative questions, an approach that has been strongly validated as being the most appropriate to a Quaker context [21]. These questions included items on spiritual life in relation to substance use (or non-use) there were also others on how the testimony was construed. Thirteen semi-structured individual tape-recorded interviews also took place, in which open-ended questions were posed about personal experience in relation to substance use, the testimonies, and spiritual life. Interview questions included, "Does alcohol/substance use have spiritual dimensions for you?" and "Do you feel that Quakerism—or Quakers-have influenced your approach or your behaviour as regards substances?...How?" 


\section{Study Findings}

The broad findings have been discussed more fully elsewhere [34,35]. In summary - and with the caveat that the small sample size restricted the generalisability of conclusions - the indications were that Quakerism has the profile of a liberal Protestant sect, in that levels of substance use were generally low and moderate. However, some latitude in behaviour that included higher levels of substance use was also found. No-one who responded to the study was in active addiction, but there were a small number of respondents who reported some level of problem in the past, and others had been affected by the addiction of a "close other".

In general terms, the null hypothesis was not upheld by the quantitative data, which showed that higher engagement tended to be related to lower use, though there were some variations in these findings. "Formal engagement" and the spiritual factors showed the strongest relationships, particularly in the Local Meeting sample, and particularly as regards the "units of alcohol consumed on a single occasion", which was the index used to indicate moderation.

The finding on the role of spirituality is significant, and was triangulated with qualitative data to investigate patterns. This paper will first summarise the findings regarding Quaker perspectives on abstinence and moderation, then consider perspectives on spirituality, and finally discuss the quantitative investigation of spirituality in greater depth.

\subsection{Quaker Perspectives on Abstinence and Moderation}

Nowhere did the questionnaire use the words "abstinence and moderation" but these were the terms that were often used in responses to the questions: "Do you think Quakerism gives clear guidelines for behaviour regarding substance use?" and "In so far as you feel there are guidelines in Quakerism, how do they affect you?"

The majority of the sample converged and described moderation and abstinence in some form [34]:

"Moderation, respect for self and others, simple life-style, these things (substances) are not needed."

"Moderation in everything."

The community aspect was valued by many.

"I like being part of a community in which it's acceptable not to drink."

However, the data also indicate that moderation was not necessarily construed as a "normative standard" in the authoritative sense found in some other denominations. This is likely to be the result of the "liberal belief culture" and its associated values. One respondent summarised that the guidelines:

"...encourage me to form a considered view of the effects of substance use on myself and others, while not being censorious towards others who are different."

This inclusiveness and non-judgmentalism was seen as positive and supportive, particularly by younger Quakers, and by those who had experienced emotional distress and/or problems with substances. 
“...I do have a firm belief that people take drink and drugs to keep away from emotions they can't deal with...the knowledge that it's not forbidden - that it's for me to make my own choices...I find that very reassuring... a real lifeline..."

"I think Quakerism is very good at that...You know, it's people saying 'I've made that decision that I no longer do that, but I'm not telling you that's right for you.' The whole thing is about not judging people and accepting."

The following inductive categories of respondents were constructed from the questionnaire responses and the qualitative interviews: (i) those who viewed Quaker guidelines as confirmatory and supportive to their own attitudes and behaviour; (ii) those who described using Quaker guidelines as an ingredient in personal judgement; (iii) those who recognised themselves departing from Quaker advice at times; (iv) those who rejected the idea of Quaker guidelines influencing their behaviour at all.

\subsubsection{The Relationships between Testimony and Behaviour}

The testimony of abstinence and moderation emerges as generally valued and "lived" for modern Quakers, which reflects the general study finding in terms of behaviour. However, comparison of respondents' behavioural data with their qualitative data revealed some interesting findings. For example, some within the group who did not endorse the idea of Quaker guidelines nevertheless acted moderately, whilst some of those taking substances at higher levels valued the idea of moderation.

One current indicated in the qualitative data was that people may have come to Quakerism in adulthood, and were already moderate. One such participant said "These decisions made before coming to Quakers". The fact that Quakerism attract some adherents who have a sympathy with the testimony is likely to be one factor in the generally low-use denominational profile.

Another strand in the qualitative data was expressed by another respondent, for example, stated, "I am so moderate in my intake only drinking alcohol occasionally that the [Quaker] guidelines are not an issue." This is congruent with the analysis of Orford in his discussions of substance problems, who describes a continuum, which at one end shows "unremarkable behaviour characterized by relatively little inclination and requiring little obvious restraint to keep it in bounds" [4]. Towards the other end of the use continuum, the qualitative data suggest that the testimony supports the exercise of some restraint for some of those who showed higher levels of use. For example, one such respondent stated, "It certainly keeps things under control a bit."

For these individuals, this process could be characterized less as attempted conformity to a normative standard than a lived dialogue with the testimony. For the small number of people who had experienced either concern about their own use, or addiction/dependency, the community norms of abstinence and moderation were experienced as reinforcing their own decisions and making them feel at home in a group that generally see low use as positive.

\subsubsection{The Community Dimension to the Interpretation of Testimony}

Community openness and support emerge as a significant part of this dialogue, particularly in the younger Quaker group: the lived testimony of moderation and abstinence includes "not being censorious 
to those who are different". The values and perspectives that underlie this approach (which was commonly expressed) were illuminated by qualitative questions in the survey completed by all participants.

One of these questions asked respondents to rank the aspects of Quakerism they personally found most fulfilling in a list of ten. Two items were chosen in first or second place by over three quarters of respondents (with the two often being chosen in combination). The first of these was "that of God in everyone", which is a central, much-valued and quoted passage from the Quaker "Advices and Queries": "Walk cheerfully over the world answering that of God in everyone." ([3], 19.32). The second item was a phrase synthesized from Quaker research for the study - "a concentration on spiritual path rather than fixed doctrines".

This general convergence on these two aspects, chosen from options that involved several other aspects of Quakerism, indicate how the letter of the testimony is informed by shared values that are perceived as being both primal and spiritual in nature. "That of God in everyone" simultaneously expresses the spiritual value of every person, a fundamental connectedness between them that is spiritual in nature, and an approach that tends away from adverse judgments based on behaviour. The second statement shows how highly spiritual seeking is valued, and that this is privileged above simple adherence to prescriptive approaches.

These perspectives relate to several of the aspects of spirituality summarised by Cook [15] — particularly relatedness, humanity, core/soul, authenticity/truth, values, and wholeness. In the Quaker sample, these were expressed as shared values as well as experiences. The sense of this data is that these fundamentally spiritual approaches_-particularly of connectedness within a spiritual whole —inform and modulate how abstinence and moderation act as a normative standard within the Quaker group.

The study findings as a whole have been related to suggestions in the literature [19,34-36] that strongly proscriptive sects may lose adherents who move away from group norms as regards substance use in an effort to reduce feelings of guilt or shame, or because the level of cognitive dissonance can become too great. The study concluded that overall, there is a balance between the letter of Quaker testimony on the one hand, and the spirit of Quaker liberalism based on respect for the inner light and that of God in everyone on the other. This balance-which needs to be held in tension-appears to check the potentially excluding effects of one, and the potentially fragmenting effects of the other.

Spiritual values emerge as an integral part of this framework, both at an individual and a community level. The ways in which spirituality is construed in relation to substance use was explored more specifically in qualitative and quantitative data, and the findings are set out below.

\subsection{Study Findings in Relation to Spirituality}

\subsubsection{Quaker Perspectives on Spirituality and Substance Use}

The specific qualitative investigation began with a questionnaire item aimed at eliciting both personal and community meanings. It asked about substance use and its effects — or potential effects — on spiritual life. In general, respondents felt that substance use was unhelpful to spiritual life. The following seven inductive categories systematise the range of perspectives that were expressed: 
(i) Mind-clouding/cognitive effects:

"I think the ability to think clearly helps with a spiritual life. I think that the use of substances can get in the way of this."

"Non-use means I am clearer in my thinking and making a choice in 'Free Will"”.

(ii) Effect on their direct relationship with God:

"The clearer my consciousness, the more alive I feel and receptive to God's wisdom."

"I don't think I could be connected to God if I was befuddled by alcohol or drugs."

(iii) Effect on the nature of the spirit:

"I think it would affect one's thinking reasoning discernment and thus one's spiritual life."

"Non-use-less clouded inner light—hopefully!"

(iv) Effect on the body and therefore the spirit:

"Spiritual life enhanced by healthy body."

"I think it can harm, and this includes caffeine and chocolate and antibiotics and steroids etc."

(v) False contentment/delusion:

"Contentment comes from within not without."

(vi) Adverse practical effects:

"Drinking has sometimes meant tiredness on Sunday morning, which affects my likelihood of going to Meeting."

(vii) Addiction as the issue:

"Keep me free from addiction."

Whilst most respondents expressed the view that substance use would be unhelpful to spiritual life, a small minority did not take this view.

"Experimental experience can sometimes enhance knowledge and therefore spiritual life."

[YFGM respondent].

In general, then, the community norms seemed to be that substance use is, or could be, unhelpful to spiritual life, though there were a minority who did not take this perspective.

\subsubsection{Quantitative Results of the Investigation into Spirituality and Substance Use}

It will be remembered that the methodology allowed the three engagement measures to be considered separately in relation to substance use. "Formal engagement" measures did show a relationship with consumption. The combined "spirituality" measure showed some of the strongest correlations with lower use behaviour. Attendance at Meeting for Worship also showed a correlation with lower frequency drinking. This suggests that both personal and shared spiritual experience may have some significance in the use/engagement relationship amongst the Quaker sample. 
As regards the detailed analysis of the three spiritual measures, all three showed correlations with lower use behaviour. The "frequency of prayer or meditation" index was most reliably correlated with lower levels of consumption, a feeling that "God's presence is available in everyday life" also showed some relationships, while the subjective rating of the importance of spirituality to the individual showed fewer relationships.

Although higher spirituality was in general associated with lower substance use, one positive correlation did emerge within the younger sample. This was found between the combined spirituality measure and more recent cannabis use. The study methodology enabled the nature of this to be examined in more detail. Closer statistical analysis showed that recent cannabis consumption was not related to the frequency of prayer and meditation, but it was positively related to the other two spiritual measures to varying degrees.

This finding was further investigated qualitatively. A variation in responses emerged. For example, one respondent said of their cannabis use, "I sometimes had that sense of connection with world or other people... and so that is where I feel there is an affinity between the basis of my spirituality which I relate to Quakerism, and substances"-while another described their experience of cannabis as "escaping" and unhelpful to a relationship with God [34].

\subsection{Interpretation of the Findings in Relation to Spirituality}

The results of the quantitative spirituality investigation were considered in relation to constructs and theories in the research literature. In one of the early papers to focus interest on this subject of spirituality and substance issues, Miller divided the larger construct of spirituality into three categories [5]:

- Behaviour: "overt behavior, such as religious and spiritual practices";

- Belief: "a sense of interrelatedness of living beings, soul or spirit, and life beyond material existence";

- Spiritual experience, in the form of "mystical or convictional experiences, serenity and oneness".

Cook's [37] definition of spirituality includes these elements in a broader way:

'It may be experienced as a relationship with that which is intimately 'inner', immanent and personal, within the self and others, and/or as relationship with that which is wholly 'other', transcendent and beyond the self. It is experienced as being of fundamental or ultimate importance..."

All of these aspects were apparent in the Quakers in the study. One questionnaire item asked respondents what spiritual awareness meant to them. A series of options were given: $60 \%$ selected "connectedness/joining with all things", while 45\% selected "the Inward Light", and 25\% transcendence (responses were not confined to one category). Similarly, understandings of God involved "the inward light" (57\%), "love" (50\%), and "spirit" (40\%). While all the elements of Cook's definition were actively mobilized, then, connectedness and the inward light were particularly salient - a point that will be returned to as the findings are analysed further. 


\subsubsection{The Roles of Spiritual Behaviour and Spiritual Awareness}

The statistical methodology of a cross-sectional study cannot prove causality, or the direction of it - so if causality exists at all, it must be recognized that higher levels/greater experience of spirituality may lead to lower levels of substance use, or that higher levels of spirituality could be the result of lower substance use. The qualitative data is suggestive in uncovering currents.

These data broadly indicate that substance use is generally seen as unhelpful to spiritual life, and this supports individuals in choosing not to engage in immoderate use. The fact that spirituality readings showed stronger (inverse) correlations with the level of alcohol use on a single occasion than with frequency of use support this suggestion.

In the detailed statistical analysis, all three spirituality measures-frequency of prayer or meditation, a sense of the availability of God, and a sense of the importance of the spiritual dimension of life - are generally associated with lower use. A common link between all these forms of spirituality and lower substance use can therefore be inferred. Spirituality emerges as a significant factor in the moderate profile of Quakers in the sample, therefore.

However, the latter two measures of spirituality did show some variation in findings, whilst the frequency of prayer or meditation showed more uniform effects. It is likely, therefore, that prayer and meditation may have a particularly powerful role in the low use/high religiosity relationship in the Quaker sample.

The reasons for this may lie partly in the framings that Miller [5] suggests. A sense of the importance of the spiritual and the availability of God relate to what Miller terms "idiographic" spirituality, which could also be described as subjective spiritual awareness (and were measured by subjective questions in this study). Qualitative data suggested that this was often linked to a sense of immanence, connectedness or transcendence.

Prayer and meditation, whilst of course based on inner experience, does have an objective, measurable dimension in terms of expression (time devoted), and was scored quantitatively by frequency in the study. Prayer and meditation can be undertaken with some measure of conscious control, and the benefits - either in terms of a relationship with the divine, or an ability to reach the "still centre" - may also increase with practice (as Groves outlines, for example [38]). The simple fact of regular time devoted to prayer and meditation may express a priority, and the practice may also allow spiritual experience to become more salient for the individual in relation to other aspects of life. All of these factors may explain the study finding that prayer and meditation have a more consistent role in mediating substance use than does idiographic spirituality. It is also notable, in the context of the larger observation that spirituality can exist independently of religion $[17,18]$, that prayer and meditation have stronger links with traditional religious practice of various kinds. This presents interesting areas for further research.

Prayer/meditation also has strong correspondences with Step 11 of the AA programme: "Sought through prayer and meditation to improve our conscious contact with God as we understood Him, praying only for knowledge of His will for us and the power to carry that out." There were too few participants in recovery within this sample for their results to affect the statistical relationships. However, the AA stress on spiritual practice as a necessary ingredient in recovery further underlines 
the study findings about the specific importance of spiritual practice within a lower use sample-and suggests, at least potentially, that spiritual practice may also have a role in resilience.

To summarise, then, the study found that in general a greater stress on, or experience of, spirituality was related to lower substance use, and this relationship was particularly strong when considering spiritual practice. The relationships involved do not have to be causal, but the quantitative and qualitative data combined suggest that spiritual practice may have a role in resilience as well as in recovery. A sense of congruence between Quaker principles and the AA spiritual programme was noted, and will be discussed further.

\subsubsection{Twelve-Step Recovery and the Quaker Community}

Quakerism's stress on the "inner light", and the centrality of spiritual experience-and its reluctance to define God within the limitations of human words - have all been analysed by Dandelion [20,39]. Quakerism's non-credal nature, the lack of a priesthood and hierarchy-and the silent method of Quaker worship, which involves "ministry", when an individual feels moved by the Inner Light to speak in worship — embody the primacy of the spiritual, and de-emphasise doctrine and fixed forms of authority. All of this has a resonance for members of Alcoholics Anonymous who come to Quakerism, who often feel a congruence between the Quaker liberal belief culture and the latitude about how the "Higher Power" is experienced within AA.

Explorations of the relationships they see between the approach of Quakerism and the AA spiritual programme can also be found elsewhere $[34,40]$. One person recounts the following experience:

"I was made to feel welcome and wanted by my local PM. ${ }^{2}$ I had at last found a place where my spirituality could flourish. I didn't have to believe, or pretend to believe, the impossible. There was no creed, no doctrine, only expectant waiting. After a few years I applied for membership and was accepted."

A similar feeling emerged from the Fellowship participants in this study:

"AA is pure Quakerism. We follow spiritual principles and a spiritual path."

"From being a dirty broken down alcoholic I prayed to Jesus to help me and as long as I remain clean and sober I can have a rich full life."

This sense of affinity between Quakerism and the Fellowships has been expressed many times at QAAD events by people who are members of both, and has also been discussed in QAAD's quarterly magazine [41].

As regards other spiritual paths to recovery within Quakerism, Cook [37] has commented that research on spirituality and recovery has mainly focused on the Fellowships. This study did discover one participant who linked their abstinence with spirituality, which was found through attending Quaker Meetings for Worship:

"Gradually after attending Meeting over the years I came to believe if there was 'that of God' in everyone there must be some in me and I wasn't all bad...”

2 Preparative Meeting was the name for a local Quaker Meeting; they are now known as Local Meetings (LMs). 


\subsubsection{Summary of the Possible Relationships between Spirituality and Use}

Spiritual values, spiritual awareness and experience, and spiritual practice have all been seen as relevant to the substance use-religiosity relationship, but pathways have still to be fully defined.

The results of this study indicate that for Quakers, spiritual awareness, the stress on spiritual experience as primal, and a sense of connectedness are particularly important and binding at a community level. Shared spiritual seeking, a sense of connectedness, and "that of God in everyone" are salient in enabling an inclusive community among Quakers. The community generally continues the testimony of moderation and abstinence, but keeps it in balance with these spiritual values, which can support those whose behaviour may differ.

For the individual, "idiographic" forms of spirituality are generally associated with lower substance use. Prayer and meditation has a more widespread association with low use, and may have a specific role in resilience within the Quaker sample. Factors that help explain why this might be the case have been discussed.

It is acknowledged, however, that another possible reading of the findings is that low substance use and prayer/meditation may not be directly and causally connected - both could be related to one or more other factors, which in their turn link with other kinds of stresses or stabilities in people's lives. This would be consistent with the qualitative data noted earlier, that a substantial minority of Quakers in the study found that moderation simply reflected their natural inclinations, while some of those using at higher levels were actively engaging with the testimony but finding it difficult. If the "other factors" explanation is valid, spirituality in general and spiritual practice in particular could be considered to have a reinforcing and strengthening role rather than a simple causal one. To put this another way, spiritual practice may help in reducing substance problems at all stages along the substance use/problem use spectrum — but this does not mean that higher levels of substance use result from lower frequency of spiritual practice.

This latter finding does present some interesting points for deliberation in religious communities. Much of the research effort on spirituality and recovery has resulted from the realisation that spirituality has been under-acknowledged in some treatment systems $[5,15]$, and research gave an evidence-base for the balance to be corrected. This has been helpful to those suffering from addiction in offering a richer and more effective programme of "care". However, there is likely to be a need for discernment if spiritual practice is to be regarded instrumentally and/or prescriptively within a religious group.

\section{Concluding Remarks}

Quakerism has a tradition of engagement with questions relating to the use of alcohol and other substances from its earliest history. Although there is no claim to distinctiveness here, a spiritual framework is at the heart of its approach to substances. The paradigm that was developed from this study [34-36] suggests that the balance between two spiritually-based values - the testimony on abstinence and moderation, and the acceptance of "that of God in everyone" within the liberal belief culture - can, at its best, provide a community that is generally moderate but not excluding those whose behaviour is different. A further benefit of this nexus of values and the liberal belief culture is 
that Quakerism is often experienced as congruent to members of Alcoholics Anonymous, and the Quaker group is further enriched by them.

All the forms of spirituality that were measured emerge as a significant element in the general low use in the Quaker sample, and the qualitative data suggest that this finding may have several patternings at the community and individual level.

One limitation on this study is that it elided prayer and meditation; separate investigation of the former may be instructive and perhaps more appropriate in other religious contexts. Limitations in terms of sample size are acknowledged, as are the inherent limitations on cross-sectional methodologies in establishing causation.

This research offers a contribution to the literature in being the only study among Quakers that relates to substance use, and in using a methodology that allowed a differentiated pattern to emerge in relation to different aspects of Quaker religiosity and spirituality. The role of spirituality as a significant factor in low use is confirmed and delineated in a way that remains unusual in the literature, and illuminates specific pathways in the relationship between religiosity, spirituality and substance use within the Quaker sample. The part prayer and meditation play in terms of resilience to substance problems is of interest, and would benefit from further study both among Quakers and elsewhere.

\section{Acknowledgments}

The supervision of P. Dandelion during the research process is gratefully acknowledged. The assistance of D. Masses was also much appreciated.

\section{Conflicts of Interest}

The author declares no conflict of interest.

\section{References and Notes}

1. John L. Nickalls. The Journal of George Fox. Edited by John L. Nickalls. Cambridge: Cambridge University Press, 1952, pp. 2-3.

2. Nick Heather, and Ian Robertson. Problem Drinking, 3rd ed. Oxford: Oxford University Press, 1997, p. 13.

3. Britain Yearly Meeting (Society of Friends). Quaker Faith and Practice: The Book of Christian Discipline of the Yearly Meeting of the Religious Society of Friends (Quakers) in Britain, 5th ed. London: The Yearly Meeting of the Religious Society of Friends (Quakers) in Britain, 2013. (N.B. The modern edition of Quaker Faith and Practice includes quotations from earlier Meetings or gatherings of Quakers from the seventeenth century onwards; this is one such quotation).

4. Jim Orford. Excessive Appetites: A Psychological View of Addictions, 2nd ed. Chichester: John Wiley and Sons, 2001.

5. William R. Miller. "Researching the Spiritual Dimensions of Alcohol and other Drug Problems." Addiction 93 (1998): 979-90.

6. John Punshon. Testimony and Tradition: Some Aspects of Quaker Spirituality. London: Quaker Home Service, 1990. 
7. Letter to The British Friend, 1851, Fourthmonth. Friends House Library, London.

8. Rose K. Goldsen, Morris Rosenberg, Robin M. Williams, and Edward A. Suchman. What College Students Think. Princeton: Van Nostrand, 1960, pp. 177-80.

9. Snell Putney, and Russell Middleton. "Dimensions and Correlates of Religious Ideologies." Social Forces 39 (1961): 285-90.

10. Steven R. Burkett, and Mervin White. "Hellfire and Delinquency: Another Look." Journal for the Scientific Study of Religion 13 (1974): 455-62.

11. Richard L. Gorsuch. "Religious Aspects of Substance Abuse and Recovery." Journal of Social Issues 51 (1995): 65-83.

12. Dale D. Chitwood, Michael L. Weiss, and Carl G. Leukefeld. "A Systematic Review of Recent Literature on Religiosity and Substance Use.” Journal of Drug Issues 38 (2008): 653-88.

13. Richard Jessor, and Shirley L. Jessor. Problem Behavior and Psychosocial Development: A Longitudinal Study of Youth. New York: Academic Press, 1977.

14. Elmer W. Bock, John K. Cochran, and Leonard Beeghley. "Moral Messages: The Relative Influence of Denomination on the Religiosity-Alcohol Relationship." The Sociological Quarterly 28 (1987): 89-103.

15. Christopher C. Cook. "Substance Misuse." In Spirituality and Psychiatry. Edited by Chrisopher Cook, Andrew Powell and Andrew Sims. Glasgow: Bell \& Bain, 2009, pp. 143, 147.

16. Theodore J. Chamberlain, and Christopher A. Hall. Realized Religion. Philadelphia: Templeton Foundation Press, 2000, pp. 118-137, 189-197.

17. Harold G. Koenig, Michael E. McCullough, and David B. Larson. Handbook of Religion and Health. New York: Oxford University Press, 2001, pp. 169-172.

18. Harold G. Koenig. Faith and Mental Health: Religious Resources for Healing. Philadelphia: Templeton Foundation Press, 2005, pp. 43-81, 109-112.

19. Kenneth Mullen, Rory Williams, and Kate Hunt. "Irish descent, Religion, and Alcohol and Tobacco Use." Addiction 91 (1996): 243-54.

20. P. Dandelion. A Sociological Analysis of the Theology of Quakers: The Silent Revolution. Lampeter: Edwin Mellen Press, 1996.

21. P. Dandelion, and Roger Homan. "Questioning Quakers.” Social Compass: International Review of Sociology of Religion 42 (1995): 487-95.

22. Alan Bryman, and Emma Bell. Business Research Methods, 3rd ed. Oxford: Oxford University Press, 2011, p. 56.

23. Martyn Hammersley. "The Relationship between Qualitative and Quantitative Research: Paradigm Loyalty versus Methodological Eclecticism." In Handbook of Research Methods for Psychology and the Social Sciences. Edited by John T. E. Richardson. Leicester: British Psychological Society Books, 1996.

24. Barney G. Glaser, and Anselm L. Strauss. The Discovery of Grounded Theory Strategies for Qualitative Research. Chicago: Aldine Publishing Company, 1967.

25. David B. Larson, Kimberly A. Sherrill, John S. Lyons, Frederic C. Craigie, Jr, Samuel B. Thielman, Mary A. Greenwold, and Susan S. Larson. "Associations between Dimensions of Religious Commitment and Mental Health Reported in the American Journal of Psychiatry and Archives of General Psychiatry: 1978-1989.” The American Journal of Psychiatry 149 (1992): 557-59. 
26. Morton B. King, and Richard A. Hunt. "Measuring the Religious Variable: A Replication." Journal for the Scientific Study of Religion 11 (1972): 240-51.

27. Dale M. Hilty. "Religious Belief, Participation and Consequences: An Exploratory and Confirmatory Analysis." Journal for the Scientific Study of Religion 27 (1988): 243-59.

28. Thomas M. Allen, and Celia C. Lo. "Religiosity, Spirituality, and Substance Abuse." Journal of Drug Issues 40 (2010): 433-59.

29. LaVerne A. Berkel, Tonya D. Armstrong, and Kevin O. Cokley. "Similarities and Differences between Religiosity and Spirituality in African American College Students: A Preliminary Investigation." Counseling and Values 49 (2004): 2-14.

30. James A. Neff. "Exploring the Dimensionality of 'Religiosity' and 'Spirituality' in the Fetzer Multidimensional Measure.” Journal for the Scientific Study of Religion 45 (2006): 449-59.

31. Lisa Pullen, Mary Anne Modrcin-Talbott, William R. West, and Robert Muenchen. "Spiritual High vs. High on Spirits: Is Religiosity related to Adolescent Alcohol and Drug Abuse?" Journal of Psychiatric and Mental Health Nursing 6 (1999): 3-8.

32. Lynn G. Underwood. "Ordinary Spiritual Experience: Qualitative Research, Interpretive Guidelines, and Population Distribution for the Daily Spiritual Experience Scale." Archive for the Psychology of Religion 28 (2006): 181-218.

33. Lynn G. Underwood. "The Daily Spiritual Experience Scale: Overview and Results." Religions 2 (2011): 29-50.

34. Helena V. Chambers. "An Investigation into the Relationships between Quaker Engagement and Substance Using and Gambling Behaviours." Ph.D. Thesis, University of Birmingham, December 2006.

35. Quaker Action on Alcohol and Drugs. To Use or Not To Use. Gosport: Ashford Colour Press, 2006.

36. Helena V. Chambers. "Modern Testimonies: The Approach of Quakers to Substance Use and Gambling." In The Quaker Condition: The Sociology of a Liberal Religion. Edited by Ben P. Dandelion and Peter Collins. Newcastle: Cambridge Scholars Publishing, 2008.

37. Christopher C. Cook. "Addiction and Spirituality." Addiction 99 (2004): 539-81.

38. Paramabandhu Groves. "Buddhist Approaches to Addiction Recovery." Religions 5 (2014): 985-1000.

39. P. Dandelion. "The Creation of Coherence: The 'Quaker double-culture' and the 'absolute perhaps'.” In The Quaker Condition: The Sociology of a Liberal Religion. Edited by P. Dandelion and Peter Collins. Newcastle: Cambridge Scholars Publishing, 2008.

40. Laurie Andrews. "Liberal Quakerism and 12-step spirituality: Realised Universalism?” Friends Quarterly, 2012 Available online: http://aaagnostica.org/wp-content/uploads/2014/08/LiberalQuakerism-and-12-Step-Spirituality.pdf (accessed on 23 February 2015).

41. Yveline Arnaud. "Pure places to practice." QAADRANT, 2014. Available online: http://www.qaad.org/wp-content/uploads/2014/12/Qaadrant-Dec-14-WEB.pdf (accessed on 24 February 2015).

(C) 2015 by the author; licensee MDPI, Basel, Switzerland. This article is an open access article distributed under the terms and conditions of the Creative Commons Attribution license (http://creativecommons.org/licenses/by/4.0/). 